\title{
Identification of MHCIl variants associated with chlamydial disease in the koala (Phascolarctos cinereus)
}

Chlamydiosis, the most common infectious disease in koalas, can cause chronic urogenital tract fibrosis and infertility. High titres of serum immunoglobulin $\mathrm{G}$ against $10 \mathrm{kDa}$ and $60 \mathrm{kDa}$ chlamydial heat-shock proteins (c-hsp10 and c-hsp60) are associated with fibrous occlusion of the koala uterus and uterine tube. Murine and human studies have identified associations between specific major histocompatibility complex class II (MHCII) alleles or genotypes, and higher c-hsp 60 antibody levels or chlamydia-associated disease and infertility. In this study, we characterised partial MHCII DAB and DBB genes in female koalas $(n=94)$ from a single geographic population, and investigated associations among antibody responses to c-hsp60 quantified by ELISA, susceptibility to chlamydial infection, or age. The identification of three candidate $\mathrm{MHCll}$ variants provides additional support for the functional role of $\mathrm{MHCll}$ in the koala, and will inform more focused future studies. This is the first study to investigate an association between MHC genes with chlamydial pathogenesis in a non-model, free-ranging species. 
1 Authors

2 Quintin Lau, Joanna E. Griffith, Damien P. Higgins

3 Faculty of Veterinary Science, McMaster Building B14, The University of Sydney, NSW, 2006, 4 Australia

5 Corresponding Author:

6 Damien P Higgins

$7 \quad$ Faculty of Veterinary Science

8 McMaster Bldg B14

9 The University of Sydney

10 NSW 2006

11 Australia

12 damien.higgins@sydney.edu.au

$13 \mathrm{Ph}$ 61-(0)2 93517130 


\section{Introduction}

15 Chlamydiosis, caused predominantly by Chlamydia pecorum and C. pneumoniae, is the most

16 common infectious disease in koalas, causing proliferative conjunctivitis and/or chronic, fibrotic

17 disease of the urogenital tract, which can lead to infertility and death (Griffith et al. 2013;

18 Obendorf 1983). Heat shock proteins (hsp) are highly conserved polypeptides produced under

19 stress conditions to preserve cellular activity, and are upregulated by Chlamydiae in their

20 persistent intermediate forms, which are induced by IFN $\gamma$ in sub-lethal Th1 immune responses

21 (Beatty et al. 1994). Evidence suggests that most pathological damage from chlamydial infections

22 results from type IV delayed-type hypersensitivity to chlamydial hsps 10 and 60, in a deleterious

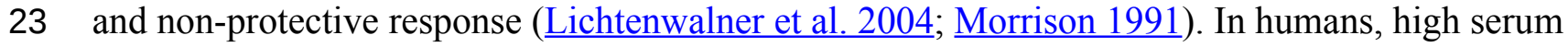

24 immunoglobulin $\mathrm{G}(\mathrm{IgG})$ titres against 10-kDa and 60-kDa chlamydial heat shock proteins (c-

25 hsp10 and c-hsp60) are associated with chronic infection, salpingeal fibrosis, tubal infertility and

26 ectopic pregnancy (Betsou et al. 1999; LaVerda et al. 2000; Toye et al. 1993; Zhong \& Brunham

27 1992); in Chlamydia-infected koalas, high anti c-hsp10 and c-hsp60 titres are also associated with

28 fibrous occlusion of the uterus and uterine tube (Higgins et al. 2005).

29 Chlamydial hsp60 antibody responses and Chlamydia-associated pathology could be genetically

30 predisposed: in mice, greater c-hsp60 antibody responses are associated with alleles of genes in

31 or near IA genes within the major histocompatibility complex (MHC) (Zhong \& Brunham 1992).

32 The murine IA genes are highly polymorphic MHCII genes that encode for membrane-bound

33 molecules that bind specific exogenous antigens and present them to T lymphocytes

34 (Balakrishnan \& Adams 1995; Kalish 1995). These are orthologous to the human HLA-DQ $\alpha$ and

$35 \beta$ genes and, similarly, two human class II alleles, DQA1*0401 and DQB1*0402 (DQ4 
36 phenotype), are associated with increased prevalence and magnitude of c-hsp60 antibody titres

37 (Gaur et al. 1999), and a class II haplotype DR8 DQ4 is associated with presence of anti c-hsp10

38 antibodies (Betsou et al. 1999). A number of studies of Chlamydia trachomatis infection in

39 women have also identified associations between HLA (MHCII) genotypes and Chlamydia-

40 associated pelvic inflammatory disease, cervicitis and tubal infertility ( $\underline{\text { Cohen et al. } 2003}$; Cohen

41 et al. 2000; Ness et al. 2004).

42 Variation in MHC genes is widely considered important for resistance against pathogens and, 43 therefore, fitness and long-term survival, based on the important role that the MHC has in the

44 host immune system, with a growing number of studies identifying associations between MHC

45 diversity, or specific alleles/variants, and disease or survival in wildlife species (Sommer 2005).

46 Examples include: specific MHC alleles of wild Soay sheep populations associated with parasite

47 resistance and juvenile survival (Paterson et al. 1998); and in water voles and red junglefowl,

48 heterozygote and homozygote individuals possessing a specific MHCII allele have higher

49 survival rates than homozygotes of another allele (Oliver \& Piertney 2012; Worley et al. 2010);

50 and increased juvenile survival in Seychelles warblers as a result of increased MHC diversity

51 and a specific allele (Brouwer et al. 2010).

52 No marsupial orthologs to the genes associated with human c-hsp60 antibody levels have been

53 identified, due to the different MHC class II gene families of marsupials (Belov et al. 2004).

54 Partial koala MHCII genes have been characterised (Jobbins et al. 2012; Lau et al. 2013), and

55 variation across Australia described (Lau et al. 2014). Though MHCII is clearly functional in the

56 koala immune system, based on its conservation of structure relative to other species (Lau et al. 
$57 \underline{2013}$ ) and demonstration of expression and up-regulation in koala lymphocytes and tissues

58 (Canfield et al. 1996; Lau et al. 2012), associations between koala MHCII and disease have not

59 yet been investigated, but the low genetic diversity of some koala populations (Lau et al. 2014)

60 makes this an area worthy of study. To open investigation of the relevance of MHCII diversity to

61 chlamydial disease in the koala, this study makes use of archived samples and data from hospital

62 admissions from a wild Chlamydia-exposed koala population to identify MHCII DA and DB $\beta$

63 variants as candidates for more targeted investigation in prospective studies. Specifically, we aim

64 to identify if there are any associations between MHCII variants and antibody responses to c-

65 hsp60; susceptibility to chlamydial infection; or age and, therefore, likelihood of survival. 


\section{Methods}

\section{Ethics statement}

68 All samples using in this study were collected in a research project approved by the University of

69 Sydney Animal Ethics Committee (permit number AEC N00/5-2009/1/4829). The project was

70 funded by the Hermon Slade Foundation.

\section{Study population}

72 This study utilised archived samples and clinical records from female koalas $(\mathrm{n}=94)$ from the

73 Port Macquarie and Hastings River district on the mid-north coast of New South Wales,

74 Australia. The samples were collected for clinical assessment of koalas following admission to

75 the Koala Hospital, Port Macquarie, between 2005 and 2011. Blood samples were collected into

76 serum, EDTA or heparin tubes and, following centrifugation, serum or plasma was aspirated and

77 surplus blood cells, plasma and serum were stored at $-20^{\circ} \mathrm{C}$. In addition, urogenital and

78 conjunctival swabs were collected for chlamydial diagnosis $(n=37)$ and stored at $-20^{\circ} \mathrm{C}$. Some

79 koalas, which were euthanased for humane reasons under Koala Hospital operating guidelines (n $80=47$ ), were subjected to necropsy and tissues collected into $10 \%$ buffered formalin for

81 histopathological confirmation for chlamydial disease and/or stored frozen at $-20^{\circ} \mathrm{C}$. DNA

82 samples were extracted from swabs, blood cells, or liver, using the DNeasy Blood and Tissue kit

83 and protocol (Qiagen, Doncaster, Australia) and then stored at $-20^{\circ} \mathrm{C}$. 
85 Based on clinical and necropsy records, koalas were categorised into three health groups.

86 Animals with urogenital swabs positive by polymerase chain reaction (PCR) amplification of ompA gene of $C$. pecorum following Higgins et al (2011), or presence of clinical signs consistent with chlamydial disease, such as cystitis, rump staining, bladder thickening or ovarian bursal cysts; $n=63)$, were classified "past infected". Remaining animals were classed as "healthy" ( $\mathrm{n}=$ 16) or, where no clinical data were available, "unknown" $(n=15)$. Koalas of "unknown" health

91 were included only in calculations investigating associations between MHCII genotypes and chlamydial hsp-60 antibody levels, and between MHCII genotype and age. The natural lifespan of female and male koalas is over 15 and 12 years of age, respectively (Martin \& Handasyde 1999). As young koalas are more likely to present to wildlife rehabilitators as a result of misadventure and trauma during dispersal, while older koalas more commonly present for diseases such as chlamydiosis (Griffith et al. 2013), koalas were divided into four age classes in order to assess if age was a confounding factor for antibody titres: young adult, AY, two to five years old $(n=15)$; mature adult, AM, five to ten years old $(n=33)$; aged adult, AA, over ten years old $(n=25)$; and age unknown AU $(n=21)$ based on an aging system utilising tooth wear

100 class (Martin 1981).

\section{MHCII genotyping of koalas}

102 Following Lau et al. (2013), koala-specific DAB and DBB exon 2 sequences were amplified in 103 all koalas by PCR, using the primers DABEx2F: 5'-ATGCCCCAAAGCACTTCAC-3',

104 DABEx2R: 5'-CGCACTRAGAAGGGCTCA-3 and DBBEx2F: 5'-

105 AGGGACATCCCAGAGGATTTCG-3', DBBEx2R: 5'-TCTTCTGTCCACCGCGAAGG-3'.

106 Forward primers were 5'-phosphorylated for enzymatic digestion of the amplicon forward strand 107 prior to one-strand conformation polymorphism (OSCP) analysis. The PCR amplifications were 
108

109

110

111

112

113 for $10 \mathrm{~min}$.

carried out in $25 \mu \mathrm{l}$ reactions with 10-30 ng of DNA, $0.32 \mu \mathrm{M}$ each primer (Sigma-Aldrich, Sydney, Australia), 1 x HotStarTaq DNA Polymerase PCR Buffer, $1 \mathrm{mM} \mathrm{MgCl}, 0.2 \mathrm{mM}$ dNTPs, and 0.5 units of HotStarTaq DNA Polymerase (Qiagen). Cycle conditions were: initial activation at $95^{\circ} \mathrm{C}$ for $15 \mathrm{~min}$, followed by 35 cycles of $40 \mathrm{~s}$ denaturation at $95^{\circ} \mathrm{C}, 40 \mathrm{~s}$ annealing at $57^{\circ} \mathrm{C}$ or $54^{\circ} \mathrm{C}$ (for $\mathrm{DAB}$ and $\mathrm{DBB}$, respectively), and $45 \mathrm{~s}$ extension at $72^{\circ} \mathrm{C}$, and a final extension at $72^{\circ} \mathrm{C}$

114 For OSCP analysis, the forward strand of PCR products was digested with Lambda exonuclease

115 (New England Biolabs, Ipswich, MA, USA) at $37^{\circ} \mathrm{C}$ for $45 \mathrm{~min}$, and the reverse strand was

116 subjected to electrophoresis in a 5\% or 10\% acrylamide gel (DAB and DBB, respectively) at

$11730 \mathrm{~W}$ for $5 \mathrm{~h}$ at $4^{\circ} \mathrm{C}$ (Lau et al. 2013). Individuals with "genotype patterns" that were identical to

118 reference animals established by Lau et al. (2013) were considered to have those genotypes, as

119 these OSCP patterns were demonstrated to have high discriminatory power in that study. Any

120 new "genotype patterns" were referred to as novel and were characterised further to identify their

121 constituent variants using direct sequencing and molecular cloning (Lau et al. 2013). DAB

122 genotype was unavailable for one individual due to limited archived sample, and was accounted

123 for in all statistical analyses.

124 ELISA for quantification of chlamydial hsp60-specific antibodies

125 Following Higgins et al. (2005), a three-stage indirect ELISA was used for quantification of c126 hsp60 antibodies. Standard sera comprised of ten doubling dilutions of a patient plasma sample 127 with high c-hsp60 antibody titres and recombinant c-hsp60 peptide antigens were those used by 128 Higgins et al. (2005) and La Verda et al. (2000). Wells of a 96-well plate (Immulon 2HB, Thermo 
129 Labsystems, Franklin, MA, USA) were allocated as test (antigen and patient sera), control

130 (patient sera, no antigen) and blank (no antigen, no sera), and incubated with $0.1 \mu \mathrm{g}$ recombinant

131 antigen or no antigen, respectively, in $100 \mu$ bicarbonate buffer $\left(\mathrm{pH} \mathrm{9.6)} \mathrm{at} 4^{\circ} \mathrm{C}\right.$ for $28 \mathrm{~h}$. Wells

132 were then washed four times with TPBS (1x PBS pH 7.6 with $0.01 \%$ Tween 20) and then

133 incubated on a $37^{\circ} \mathrm{C}$ water bath, with $200 \mu 11 \%$ casein (Sigma, Castle Hill, Australia) in PBS

134 (120 min), followed by $100 \mu 1$ patient sera diluted 1/100 in TPBS, or $100 \mu 1$ standard sera or 100

$135 \mu 1$ TPBS for blank wells (60 min), followed by $100 \mu 1$ rabbit anti-kangaroo IgG (Bethyl

136 Laboratories, Inc., Montgomery, TX, USA) 1/50 in TPBS (60 min), then $100 \mu 1$ alkaline

137 phosphatise-conjugated donkey anti-rabbit Ig (Chemicon International, Inc., Billerica, MA, USA)

138 diluted 1/1200 in TPBS (60 min). Between incubations, wells were washed four times with TPBS

139 and, after the final incubation, with TBS (tris-buffered saline). To create a signal, wells were

140 incubated with $100 \mu \mathrm{l}$ p-nitrophenylphosphate (pNpp, Bio-Rad, Hercules, CA, USA) at room

141 temperature and the reaction was stopped at 6 min using $50 \mu 12 \mathrm{M} \mathrm{NaOH}$. Absorbance relative to

142 blank wells was read at $405 \mathrm{~nm}$ on a Spectromax 240 automated plate reader (Molecular

143 Devinces, Sunnyvale, CA, USA). Optical densities (OD) were calculated by mean differences

144 between ELISA test and control wells. To account for inter-assay variation, all OD were

145 converted to arbitrary standard units (SU) based on a standard curve generated in ReaderFit

146 Online Version (Hitachi Solutions America) with a four-parameter log-logit equation. For

147 parametric statistics, anti-hsp IgG SU results were $\log (\mathrm{SU}+0.01)$ transformed, now referred to 148 as $\log \mathrm{SU}$.

149 Statistical analysis 
150 Using general linear regression in Genstat version 14, c-hsp60 antibody levels (logSU, $n=94$,

151 d.f. $=2$ ) were compared among different age and health (infection status) groups. Following this,

152 MHC variants were evaluated with age class and health status for statistical association with

$153 \operatorname{logSU}$. Only MHC variants found in $10 \%$ to $90 \%$ of koalas studied were analysed, and non-

154 significant MHC variants were dropped from the linear regression by a stepwise procedure $(\mathrm{p}<$

155 0.05). All diagnostic plots of residuals were checked for normal distribution in Genstat. We

156 systematically used R ( R Development Core Team 2012) for Fisher's exact test to compare variant

157 presence with (i) age class, (ii) chlamydial disease ("past infected" and "healthy"), and (iii) c-

158 hsp60 antibody seropositivity: "healthy" koalas in the AY age class were used to determine a

159 limit for c-hsp60 antibody seropositivity (two standard-deviations above the mean $\operatorname{logSU}$ ) of

1601.785 , whereby all koalas were classified as c-hsp60 antibody seropositive if $\operatorname{logSU} \geq 1.785$. In

161 order to be inclusive when screening for potential genetic candidates for future, more targeted

162 studies, a less conservative modified false discovery rate (FDR) method (Benjamini \& Yekutieli

163 2001; Narum 2006) was used to determine critical significance values.

\section{Results}

165 MHCII DAB and DBB variants

166 From the 94 koalas, a total of eight DAB (DAB*10, 15, 18, 19, $21-24$, GenBank accession

167 numbers JX109927-JX109934) and four DBB variants (DBB*01 - 04, GenBank accession

168 numbers JX109922-JX109925) were identified, all of which have been described previously in

169 this population (Lau et al. 2014). Also consistent with Lau et al (2014), koalas had four to five

$170 \mathrm{DAB}$ variants and one to two DBB variants identified per individual, and the DAB*19 and

$171 \mathrm{DAB}^{*} 21$ variants were found in a majority of koalas ( $96.8 \%$ and $98.9 \%$, respectively), and were 
172 therefore not included in analyses. Although three new "genotype patterns" were identified from

173 OSCP analyses, sequencing of these patterns showed that they comprised new combinations of

174 previously identified MHCII variants.

175 Association of c-hsp60 antibody levels with age class and chlamydial infection

176 Both age class and evidence of chlamydial infection were significantly associated with c-hsp60

177 antibody levels (general linear regression). Koalas over five years of age had significantly higher

178 c-hsp60 antibody titres (logSU predicted mean \pm standard error; AM: $1.20 \pm 0.28$, AA: $0.97 \pm$

$1790.31)$ than young adults aged between two and five years old $(-0.77 \pm 0.42)(p=0.001$, least

180 significant difference at 5\% level $(1$. s.d. $)=1.03)$ (Figure 1). "Past infected" koalas had

181 significantly higher mean $\operatorname{logSU}$ values $(0.94 \pm 0.20)$ than "healthy" koalas $(-0.16 \pm 0.41)(\mathrm{p}=$

1820.019 , l.s.d. $=0.90)($ Figure 1). Age class and evidence of chlamydial infection were, therefore, 183 incorporated into regression analysis for assessing MHCII variants as predictors.

184 MHCII variants associated with chlamydial infection, c-hsp60 antibody levels and age

185 Of the four PhciDAB and four PhciDBB variants found in $10-90 \%$ of koalas studied, three

186 were associated, each with a different aspect of the pathogenesis of chlamydial disease or health

187 in the koala: $\mathrm{DBB}^{*} 04$ with antibody production, $\mathrm{DAB} * 10$ with infection and persistence, and

188 DBB*02 with age. For each group of koalas with the same MHCII variant, Fisher's exact test

189 found no differences in the proportion of c-hsp60 antibody seropositive $(\operatorname{logSU}>1.785)$ and

190 seronegative $(\operatorname{logSU}<1.785)$ individuals $(\mathrm{p}=0.158-1.000$, Supplementary material A).

191 However, general linear regression analysis showed that 
192 DBB*04-positive koalas $(1.74 \pm 0.41, \mathrm{n}=14)$ had significantly higher mean c-hsp60 antibody

$193\left(\operatorname{logSU} \pm\right.$ s.e.) values than $\mathrm{DBB}^{*} 04$-negative koalas $(0.52 \pm 0.17, \mathrm{n}=80, \mathrm{p}=0.008,1 . \mathrm{s} . \mathrm{d}=0.88)$

194 (Figure 2, Supplementary material B).

195 Association of the other two MHCII variants with infection (DAB*10) and age (DBB*02) were

196 identified using Fisher's exact tests. Based on modified FDR, the critical value for the total

197 number of hypothesis tests performed $(k=8)$ is $\mathrm{p}=0.018$ (Benjamini \& Yekutieli 2001; Narum

198 2006). On this basis, there was a strong trend for a greater percentage of "past-infected" koalas

$199(87.3 \%)$ to be DAB*10-positive than "healthy" koalas $(60.0 \%)(p=0.021$, Table 1$)$. When

200 comparing the three age classes, the proportion of DAB*15 and DBB*02-positive koalas

201 appeared to increase with age, and DBB*02 closely approached the critical significance value (p $202=0.019$ ); prevalence of the $\mathrm{DBB}^{*} 02$ variant increased from $73.3 \%$ of koalas below five years of 203 age, to $87.9 \%$ of middle aged koalas, to $100 \%$ of koalas above ten years of age (Table 2 ).

\section{Discussion}

205 Studies in mice and humans have indicated that antibody responses to chlamydial-hsp60 are 206 genetically predisposed (Gaur et al. 1999; Zhong \& Brunham 1992), but this is the first study to 207 investigate associations between MHC and c-hsp60 antibodies and chlamydial disease in a free208 living wildlife species, the koala. We identified three candidate MHCII variants; each associated 209 with one of infection $(\mathrm{DAB} * 10)$, serologic response $(\mathrm{DBB} * 04)$, or age of presentation $(\mathrm{DBB} * 02)$. 210 This is consistent with the complex pathogenesis of chlamydial disease and, indeed, parallels the 211 identification of different alleles associated with specific aspects of chlamydial disease in women 212 (Betsou et al. 1999; Cohen et al. 2000; Gaur et al. 1999; Kinnunen et al. 2002). 
213 The identification of a higher proportion of Chlamydia-infected koalas carrying the DAB*10

214 variant, relative to "healthy" koalas, is similar to studies in women where a positive association

215 was identified between specific DQ alleles and C. trachomatis infection or chlamydial cervicitis

216 (Cohen et al. 2000; Ness et al. 2004). If DAB*10 is a susceptibility variant for infection, it may

217 act by displacing another protective variant, such as the phylogenetically similar DAB*15.

218 Although there is only a single amino acid substitution between these two variants, minor

219 changes in MHC amino acid sequences can alter the pattern of epitopes that are presented (Bill et

220 al. 2005; Mealey et al. 2006), thus preventing successful binding to antigenic peptides.

221 Candidates for these peptides might include epitopes of the major outer membrane protein

222 (MOMP), which is required for cellular adhesion and for which particular epitopes are recognised

223 to elicit the Th1 response important for protective immunity against chlamydial organisms (Girjes

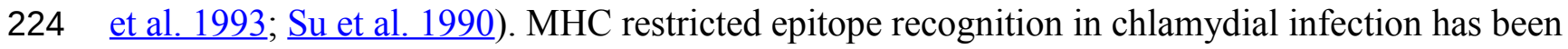

225 documented in mice, whereby a specific C. trachomatis MOMP Th cell epitope was not

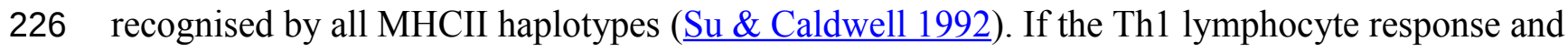

227 the associated IFN $\gamma$ secretion is limited in infected DAB*10-positive koalas, it may result in the

228 failure to eliminate the organism (Morrison \& Caldwell 2002). Further elucidation of the role of

$229 \mathrm{DAB} * 10$ could include the investigation of antigenic peptides recognised by the adaptive

230 immunity of DAB*10-positive and -negative koalas.

231 A positive association between an MHCII variant (DBB*04) and c-hsp60 antibody levels

232 suggests that this variant recognises and binds particularly well to c-hsp60 epitopes to trigger a

233 strong c-hsp specific humoral immune response. Whether DBB*04 is associated with

234 pathogenesis is less clear, and investigation of this would need to follow several possible 
mechanisms. It would be interesting to determine whether this variant triggers a cellular response,

236 which might induce type IV-associated pathology, or whether it favours a Th2 response, which

237 might induce pathogenesis by allowing chlamydial persistence. Whether $\mathrm{DBB} * 04$-positive koalas

238 produce a greater cellular immune response to hsp60 might be investigated using in vitro assays,

239 similar to those used by Kinnunen et al. (2002) to identify in women links between

240 lymphoproliferative response to chlamydial hsp60 and HLA class II variants. In addition,

241 elucidation of the Th1/Th2 balance could be gained from the study of the cytokine profile of

242 these koalas (Maher et al. 2014).

243 If the apparent increase in proportion of $\mathrm{DBB}^{*} 02$-positive koalas with age is representative of a

244 trend in the source population, it might be due to enhanced survival of animals with $\mathrm{DBB}^{*} 02$,

245 while the opposite would be indicated if this variant is only age-biased in the hospitalised

246 population. While young koalas are admitted for conditions predominantly associated with

247 dispersal, aged koalas are much more commonly admitted with chlamydial disease (Griffith et al.

248 2013) and this was evident in animals in this study. While DBB*02 is not likely to be a

249 susceptibility variant due to its high prevalence in the population, studies in other species have

250 identified a single susceptibility allele (variant) that is masked by other alleles in heterozygotes

251 (Oliver \& Piertney 2012; Worley et al. 2010) and this might permit the persistence of a

252 detrimental allele. There is no evidence to support this in the current study, as there was no

253 change in the proportion of $\mathrm{DBB}^{*} 02$ homozygotes with age (Table 2 ). It would be of interest to

254 examine the relationship between variant prevalence and age in the source (free-ranging)

255 population. 
256 Since MHC regions generally have high linkage disequilibrium and can disperse across the

257 genome (Belov et al. 2006; Siddle et al. 2009), we cannot rule out the possibility that the

258 associations found in this study instead relate to a gene linked to these MHCII candidate variants.

259 Further characterisation of the koala genome and the genetic location of the MHCII candidate

260 genes would provide insights into this. Due to the complex and multi-factorial nature of wildlife

261 disease, and difficulty in accessing and assessing free-ranging animals, studies relating risk

262 factors to disease in wildlife are very challenging. The presence of archived material was a

263 unique opportunity to begin investigations of the significance of MHCII genetics on the most

264 important infectious disease of koalas but, as with most retrospective studies, had some

265 limitations. Greater consistency in detecting and speciating chlamydial infection, such as with

266 species-specific real-time PCR (Govendir et al. 2012), and more consistent clinical classification

267 of animals, would be desirable and possible with a prospective study. Chlamydia is an extremely

268 challenging disease for this type of study, due to its chronicity, the cryptic nature of some of its

269 pathological changes, and its complex pathogenesis; and the cross-sectional sampling of

270 hospitalised koalas, which are biased for age and disease (Griffith et al. 2013), rather than

271 longitudinal sampling of a free-ranging population, limits some conclusions. Incorporation of

272 MHCII genetics in a longitudinal epidemiological study of a free-ranging population would be

273 ideal; to remove biases, provide a more thorough history of disease progression, and permit more

274 detailed survival studies. Extending studies on a wider spatial scale would also be important, to

275 investigate population-specific variants, and the significance of MHCII to disease in the southern

276 state of Victoria where most populations sampled to date are monomorphic for DBB*02 (Lau et

277 al. 2014). Studies on a wider temporal and spatial scale will require high throughput genotyping

278 methods and the present study provides the basis for a targeted approach to genotyping, for

279 example through variant-specific primers based on the candidate variants we have identified. 
281 In this study we have identified three MHCII variants in the koala that are associated with either 282 chlamydial hsp60 antibody levels (DBB*04), chlamydial infection (DAB*10), or age (DBB*02), 283 providing further support for a significant role of MHCII in the koala, and providing candidates

284 for more focused prospective studies in the future. Stemming from this study, many questions are 285 raised about the complex interaction between $\mathrm{MHC}$ and chlamydiosis, including the genetic 286 impact on the Th1/Th2 balance, recognition of specific chlamydial antigens, and resistance or 287 susceptibility to disease. This work provides the foundation and impetus for future work through 288 further characterisation of the role of these candidate variants.

\section{Acknowledgements}

290 We thank the Koala Hospital (Port Macquarie) staff and volunteers, especially Cheyne Flanagan, 291 for assistance with sample collection, and Peter Thomson for assistance with statistical analyses. 
293

294

295

296

297

298

299

300

301

302

303

304

305

306

307

308

309

310

311

312

313

314

315

316

317

318

319

320

321

322

323

324

325

326

327

328

329

330

331

332

333

334

335

336

337

338
Balakrishnan K, and Adams LE. 1995. The role of the lymphocyte in an immune response. Immunological Investigations 24:233-244.

Beatty WL, Byrne GI, and Morrison RP. 1994. Repeated and persistent infection with Chlamydia and the development of chronic inflammation and disease. Trends in Microbiology 2:9498.

Belov K, Deakin JE, Papenfuss AT, Baker ML, Melman SD, Siddle HV, Gouin N, Goode DL, Sargeant TJ, Robinson MD et al. . 2006. Reconstructing an ancestral mammalian immune supercomplex from a marsupial major histocompatibility complex. PLoS Biology 4:e46.

Belov K, Lam MK-P, and Colgan DJ. 2004. Marsupial MHC class II $\beta$ genes are not orthologous to the eutherian $\beta$ gene families. Journal of Heredity 95:338-345.

Benjamini Y, and Yekutieli D. 2001. The control of the false discovery rate in multiple testing under dependency. The Annals of Statistics 29:1165-1188.

Betsou F, Sueur JM, and Orfila J. 1999. Serological investigation of Chlamydia trachomatis heat shock protein 10. Infection and Immunity 67:5243-5246.

Bill JR, Mack DG, Falta MT, Maier LA, Sullivan AK, Joslin FG, Martin AK, Freed BM, Kotzin BL, and Fontenot AP. 2005. Beryllium presentation to CD4+ T cells is dependent on a single amino acid residue of the MHC class II $\beta$-chain. The Journal of Immunology 175:7029-7037.

Brouwer L, Barr I, Van De Pol M, Burke T, Komdeur JAN, and Richardson DS. 2010. MHCdependent survival in a wild population: evidence for hidden genetic benefits gained through extra-pair fertilizations. Molecular Ecology 19:3444-3455.

Canfield PJ, Hemsley S, and Connolly J. 1996. Histological and immunohistological study of the developing and involuting superficial cervical thymus in the koala (Phascolarctos cinereus). Journal of Anatomy 189:159-169.

Cohen CR, Gichui J, Rukaria R, Sinei SS, Gaur LK, and Brunham RC. 2003. Immunogenetic correlates for Chlamydia trachomatis-associated tubal infertility. Obstetrics \& Gynecology 101:438-444.

Cohen CR, Sinei SS, Bukusi EA, Bwayo JJ, Holmes KK, and Brunham RC. 2000. Human leukocyte antigen class II DQ alleles associated with Chlamydia trachomatis tubal infertility. Obstetrics \& Gynecology 95:72-77.

Gaur LK, Peeling RW, Cheang M, Kimani J, Bwayo J, Plummer F, and Brunham RC. 1999. Association of Chlamydia trachomatis heat-shock protein 60 antibody and HLA class II DQ alleles. Journal of Infectious Diseases 180:234-237.

Girjes AA, Ellis WAH, Carrick FN, and Lavin MF. 1993. Some aspects of the immune response of koalas (Phascolarctos cinereus) and in vitro neutralization of Chlamydia psittaci (koala strains). FEMS Immunology and Medical Microbiology 6:21-30.

Govendir M, Hanger J, Loader JJ, Kimble B, Griffith JE, Black LA, Krockenberger MB, and Higgins DP. 2012. Plasma concentrations of chloramphenicol after subcutaneous administration to koalas (Phascolarctos cinereus) with chlamydiosis. Journal of Veterinary Pharmacology and Therapeutics 35:147-154.

Griffith JE, Dhand NK, Krockenberger MB, and Higgins DP. 2013. A retrospective study of admission trends of koalas to a rehabilitation facility over thirty years. Journal of Wildlife Diseases 49:18-28.

Higgins DP, Beninati T, Meek M, Irish J, and Griffith JE. 2011. Within-population diversity of koala Chlamydophila pecorum at ompA VD1-VD3 and the ORF663 hypothetical gene. Veterinary Microbiology 156:353-358. 
339

340

341

342

343

344

345

346

347

348

349

350

351

352

353

354

355

356

357

358

359

360

361

362

363

364

365

366

367

368

369

370

371

372

373

374

375

376

377

378

379

380

381

382

383

384

385

386

387

Higgins DP, Hemsley S, and Canfield PJ. 2005. Association of uterine and salpingeal fibrosis with chlamydial hsp60 and hsp10 antigen-specific antibodies in Chlamydia-infected koalas. Clinical and Diagnostic Laboratory Immunology 12:632-639.

Jobbins SE, Sanderson CE, Griffith JE, Krockenberger MB, Belov K, and Higgins DP. 2012. Diversity of MHC class II DAB1 in the koala (Phascolarctos cinereus). Australian Journal of Zoology 60:1-9.

Kalish RS. 1995. Antigen processing: The gateway to the immune response. Journal of the American Academy of Dermatology 32:640-652.

Kinnunen AH, Surcel HM, Lehtinen M, Karhukorpi J, Tiitinen A, Halttunen M, Bloigu A, Morrison RP, Karttunen R, and Paavonen J. 2002. HLA DQ alleles and interleukin-10 polymorphism associated with Chlamydia trachomatis-related tubal factor infertility: a case-control study. Human Reproduction (Oxford) 17:2073-2078.

Lau Q, Canfield PJ, and Higgins DP. 2012. Expression and in vitro upregulation of MHCII in koala lymphocytes. Veterinary Immunology and Immunopathology 147:35-43.

Lau Q, Jaratlerdsiri W, Griffith JE, Gongora J, and Higgins DP. 2014. MHC class II diversity of koala (Phascolarctos cinereus) populations across their range. Heredity.

Lau Q, Jobbins SE, Belov K, and Higgins DP. 2013. Characterisation of four major histocompatibility complex class II genes of the koala (Phascolarctos cinereus). Immunogenetics 65:37-46.

LaVerda D, Albanese LN, Ruther PE, Morrison SG, Morrison RP, Ault KA, and Byrne GI. 2000. Seroreactivity to Chlamydia trachomatis Hsp10 correlates with severity of human genital tract disease. Infection and Immunity 68:303-309.

Lichtenwalner AB, Patton DL, Van Voorhis WC, Sweeney YTC, and Kuo C-C. 2004. Heat shock protein 60 is the major antigen which stimulates delayed-type hypersensitivity reaction in the macaque model of Chlamydia trachomatis salpingitis. Infection and Immunity 72:1159-1161.

Maher Iona E, Griffith Joanna E, Lau Q, Reeves T, and Higgins Damien P. 2014. Expression profiles of the immune genes CD4, CD8 $\beta$, IFN $\gamma$, IL-4, IL-6 and IL-10 in mitogenstimulated koala lymphocytes (Phascolarctos cinereus) by qRT-PCR. PeerJ 2:e280.

Martin R. 1981. Age-specific fertility in three populations of the koala, Phascolarctos cinereus Goldfuss, in Victoria. Wildlife Research 8:275-283.

Martin R, and Handasyde KA. 1999. The Koala: Natural history, conservation and management. Sydney: University of New South Wales Press Ltd.

Mealey RH, Lee J-H, Leib SR, Littke MH, and McGuire TC. 2006. A single amino acid difference within the $\alpha-2$ domain of two naturally occurring equine MHC class I molecules alters the recognition of gag and rev epitopes by Equine Infectious Anemia virus-specific CTL. The Journal of Immunology 177:7377-7390.

Morrison RP. 1991. Chlamydial hsp60 and the immunopathogenesis of chlamydial disease. Seminars in Immunology 3:25-33.

Morrison RP, and Caldwell HD. 2002. Immunity to murine chlamydial genital infection. Infection and Immunity 70:2741-2751.

Narum SR. 2006. Beyond Bonferroni: less conservative analyses for conservation genetics. Conservation Genetics 7:811-811.

Ness RB, Brunham RC, Shen C, and Bass DC. 2004. Associations among human leukocyte antigen (HLA) class II DQ variants, bacterial sexually transmitted diseases, endometritis, and fertility among women with clinical pelvic inflammatory disease. Sexually Transmitted Diseases 31:301-304.

Obendorf DL. 1983. Causes of mortality and morbidity of wild koalas, Phascolarctos cinereus (Goldfuss), in Victoria, Australia. Journal of Wildlife Diseases 19:123-131.

PeerJ reviewing PDF | (v2014:03:1756:1:0:NEW 26 May 2014) 
388

389

390

391

392

393

394

395

396

397

398

399

400

401

402

403

404

405

406

407

408

409

410

411
Oliver MK, and Piertney SB. 2012. Selection maintains MHC diversity through a natural population bottleneck. Molecular Biology and Evolution 29:1713-1720.

Paterson S, Wilson K, and Pemberton JM. 1998. Major histocompatibility complex variation associated with juvenile survival and parasite resistance in a large unmanaged ungulate population (Ovis aries L.). Evolution 95.

R Development Core Team. 2012. R: A language and environment for statistical computing. $R$ Foundation for Statistical Computing. Vienna, Austria: http://www.R-project.org/.

Siddle HV, Deakin JE, Coggill P, Hart E, Cheng Y, Wong ES, Harrow J, Beck S, and Belov K. 2009. MHC-linked and un-linked class I genes in the wallaby. BMC Genomics 10:310.

Sommer S. 2005. The importance of immune gene variability (MHC) in evolutionary ecology and conservation. Frontiers in Zoology 2.

$\mathrm{Su} \mathrm{H}$, and Caldwell HD. 1992. Immunogenicity of a chimeric peptide corresponding to T helper and B cell epitopes of the Chlamydia trachomatis major outer membrane protein. The Journal of Experimental Medicine 175:227-235.

$\mathrm{Su}$ H, Morrison RP, Watkins NG, and Caldwell HD. 1990. Identification and characterization of T helper cell epitopes of the major outer membrane protein of Chlamydia trachomatis. The Journal of experimental medicine 172:203-212.

Toye B, Laferriere C, Claman P, Jessamine P, and Peeling R. 1993. Association between antibody to the chlamydial heat-shock protein and tubal infertility. Journal of Infectious Diseases 168:1236-1240.

Worley K, Collet J, Spurgin LG, Cornwallis C, Pizzari T, and Richardson DS. 2010. MHC heterozygosity and survival in red junglefowl. Molecular Ecology 19:3064-3075.

Zhong G, and Brunham RC. 1992. Antibody responses to the chlamydial heat shock proteins hsp60 and hsp70 are H-2 linked. Infection and Immunity 60:3143-3149. 


\section{Table 1 (on next page)}

Table 1

Frequency of DAB and DBB variants in "healthy" koalas and "past infected" koalas. 
Table 1. Frequency of DAB and DBB variants in "healthy" koalas and "past infected" koalas.

\begin{tabular}{llll}
\hline Variant & $\begin{array}{l}\text { Proportion “healthy" } \\
(\%)\end{array}$ & $\begin{array}{l}\text { Proportion "past } \\
\text { infected" }(\%)\end{array}$ & $\begin{array}{l}\text { Fisher's exact } \\
\text { p-value }\end{array}$ \\
\hline DAB*10 & $9 / 15(60.0 \%)$ & $55 / 63(87.3 \%)$ & $\mathbf{0 . 0 2 3}$ \\
DAB*15 & $3 / 15(20.0 \%)$ & $10 / 63(15.9 \%)$ & 0.707 \\
DAB*22 & $4 / 15(26.7 \%)$ & $14 / 63(22.2 \%)$ & 0.739 \\
DAB*24 & $6 / 15(40.0 \%)$ & $14 / 63(22.2 \%)$ & 0.192 \\
DBB*01 & $1 / 16(6.3 \%)$ & $8 / 63(12.7 \%)$ & 0.678 \\
DBB*02 & $13 / 16(81.3 \%)$ & $56 / 63(88.9 \%)$ & 0.425 \\
$\mathrm{DBB}^{*} 03$ & $6 / 16(37.5 \%)$ & $21 / 63(33.3 \%)$ & 0.774 \\
$\mathrm{DBB}^{*} 04$ & $4 / 16(25.0 \%)$ & $7 / 63(11.1 \%)$ & 0.219 \\
\hline
\end{tabular}

Fisher's exact $p$-values close to the critical significance value of $p=0.019$ are in bold 


\section{Table 2 (on next page)}

Table 2

Frequency of DAB and DBB variants in koalas in each of three age classes. 
Table 2. Frequency of DAB and DBB variants in koalas in each of three age classes.

\begin{tabular}{lllll}
\hline Variant & Proportion AY (\%) & $\begin{array}{l}\text { Proportion AM } \\
(\%)\end{array}$ & $\begin{array}{l}\text { Proportion AA } \\
(\%)\end{array}$ & $\begin{array}{l}\text { Fisher's exact } \\
\text {-value }\end{array}$ \\
\hline DAB*10 & $12 / 15(80.0 \%)$ & $25 / 33(75.8 \%)$ & $21 / 25(84.0 \%)$ & 0.812 \\
DAB*15 & $1 / 15(6.7 \%)$ & $3 / 33(9.1 \%)$ & $7 / 25(28.0 \%)$ & 0.130 \\
DAB*22 & $3 / 15(20.0 \%)$ & $8 / 33(24.2 \%)$ & $7 / 25(28.0 \%)$ & 0.883 \\
DAB*24 & $1 / 15(6.7 \%)$ & $9 / 33(27.3 \%)$ & $7 / 25(28.0 \%)$ & 0.265 \\
DBB*01 & $1 / 15(6.7 \%)$ & $5 / 33(15.2 \%)$ & $2 / 25(8.0 \%)$ & 0.701 \\
DBB*02 & $11 / 15(73.3 \%)$ & $29 / 33(87.9 \%)$ & $25 / 25(100 \%)$ & $\mathbf{0 . 0 1 9}$ \\
DBB*02 & $7 / 15(46.7 \%)$ & $20 / 33(60.6 \%)$ & $11 / 25(44.0 \%)$ & 0.442 \\
homozygote & & & & \\
DBB*03 & $6 / 15(40.0 \%)$ & $10 / 33(30.3 \%)$ & $8 / 25(32.0 \%)$ & 0.765 \\
DBB*04 & $4 / 15(26.7 \%)$ & $1 / 33(3.0 \%)$ & $4 / 25(16.0 \%)$ & 0.048 \\
\hline
\end{tabular}

Fisher's exact $p$-values close to the critical significance value of $p=0.019$ are in bold. AY young adult, AM mature adult, AA aged adult. 


\section{Figure 1}

Figure 1

Mean chlamydial heat-shock protein 60 antibody levels in koalas of three health groups:

unknown $(U)$, "healthy" $(H)$ and "past infected" koalas $(I)$ and age classes: aged adult (AA), mature adult (AM), young adult (AY) and unknown (AU).

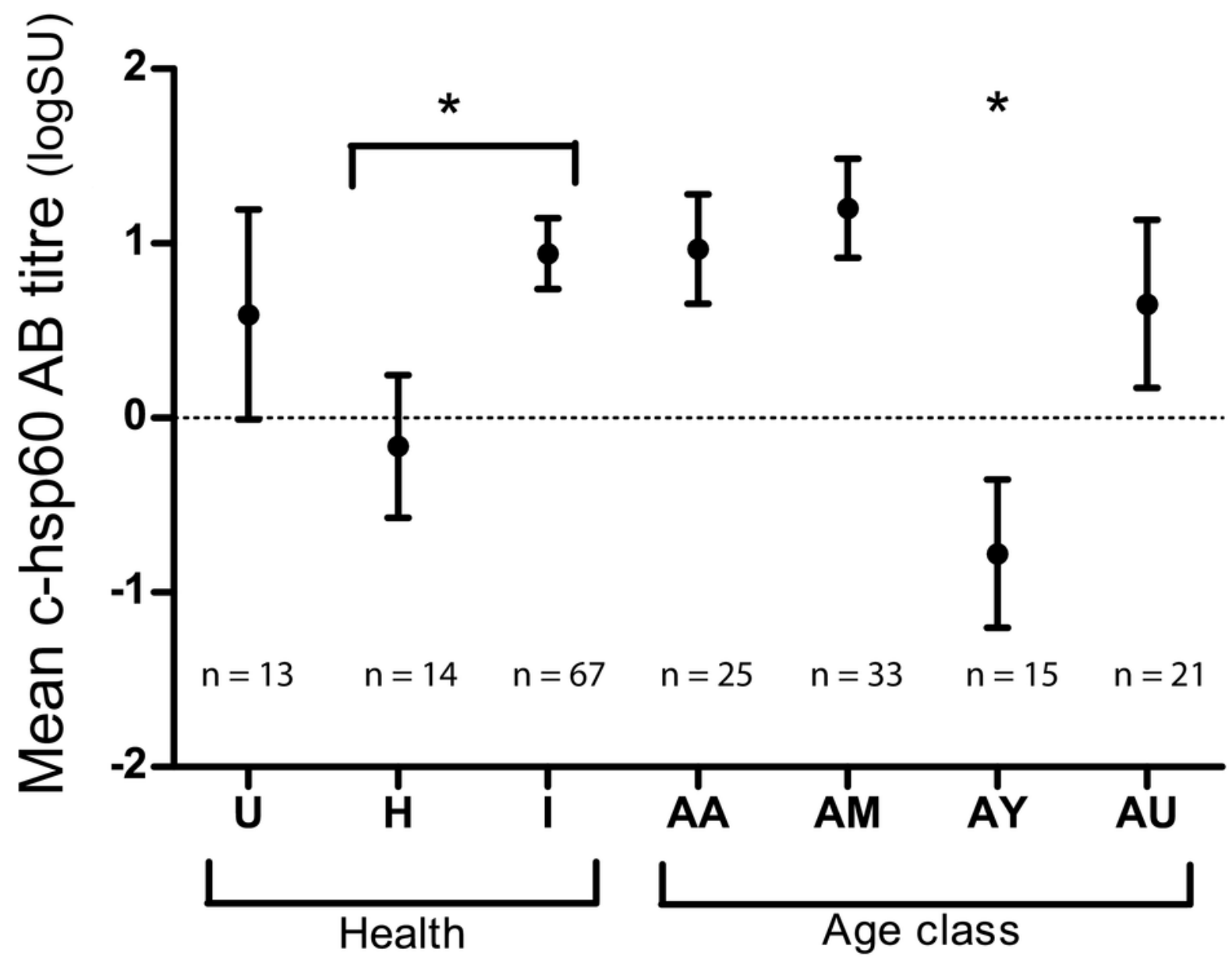




\section{Figure 2}

Figure 2

Mean log anti-chlamydial heat shock protein 60 antibody levels of koalas with (+, blue) and without (-, red) DAB and DBB variants that occurred at between $10 \%$ and $90 \%$ prevalence in the population studied.

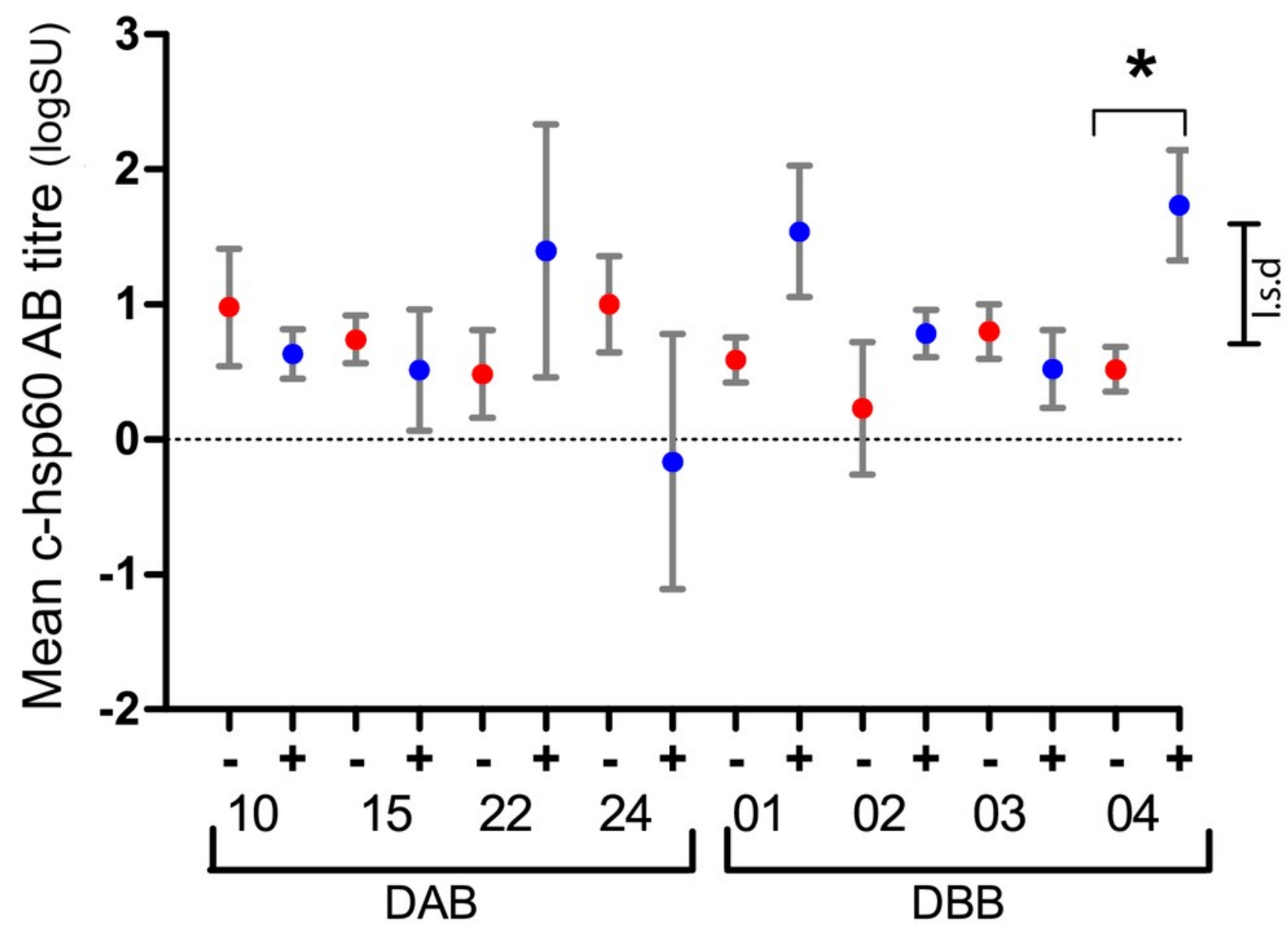

Allele 